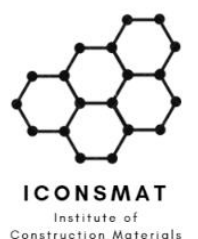

Construction Materials
Content list available at ICONSMAT

Journal of Construction Materials

Journal homepage: www.iconsmat.com.au/publication
Article history:

Received 1 January 2021

Received in revised form

17 March 2021

Accepted 25 March 2021

Available online

10 April 2021

\title{
Analysis of wastewater disposal in depleted tight gas reservoirs: A sustainable resources approach
}

\author{
Amin Taghavinejad ${ }^{1,2}$, Sepideh Shafeie*3, Armin Shirbazo ${ }^{1}$ \\ ${ }^{1}$ Department of Petroleum Engineering, Amirkabir University of Technology (Tehran Polytechnic), Tehran, Iran \\ ${ }^{2}$ Ahvaz Center for Petroleum Research, Ahvaz Faculty of Petroleum, Petroleum University of Technology, Ahvaz, \\ Iran \\ ${ }^{3}$ Faculty of Engineering, Science and Research Branch of Islamic Azad University, Tehran, Iran \\ *Corresponding author: Sepideh Shafeie, PhD Candidate, Faculty of Engineering, Science and Research Branch of \\ Islamic Azad University, Tehran, Iran. E: Sepidehshafeie@yahoo.com
}

\begin{abstract}
Wastewater injection into oil and gas fields are implemented for various purposes via injection wells. Disposing wastewater, which is mostly waste saltwater produced with hydrocarbons in oil and gas fields, into underground petroleum reservoirs are usually tied with environmental purposes. Injection of wastewater into geologic strata may encompass different applications: hazardous or non-hazardous wastewater disposal, enhanced recovery from petroleum reservoirs or merely wastewater storage. Aside from the purpose of wastewater injection, modeling of wastewater flow in porous media of underground rock strata can be challenging in different petroleum reservoirs and wells. In this study, a tight gas reservoir (TGR) - as a large-scale sustainable material to store wastewater-is considered to be studied for water disposal via a multistage fractured horizontal well (MSFHW) by numerical simulation. Host rock layer is considered to be initially saturated with low-pressure methane gas and water injection has to be performed through the hydraulic fractures of the MSFHW into the rock pore volume. Injection is performed under constant bottomhole flowing pressure and several sensitivity analyses are investigated to outline important rock characteristics in TGRs affecting performance of wastewater injection into them.
\end{abstract}

DOI: 10.36756/JCM.si1.4 (C2021 Institute of Construction Materials

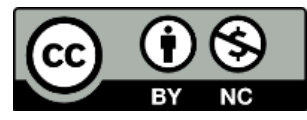

Keywords: Wastewater disposal, Tight gas reservoir, Water injection, Reservoir simulation, Sustainable resources 


\section{Introduction}

Nowadays, activists in different sciences and fields of studies try their best to cover all goals of sustainable development. In order to reach this goal, they use the optimum methods and modern knowledge in all steps of projects. Accordingly, paying attention to environmental aspects in each part of work is a necessary point. Industrial experts, based on their crucial role in development, are effective players in this area. Thus, they put their attempt on answering various kinds of issues all around the world considering environmental and economic concerns [1]. These efforts include any new optimized method which is helpful in order to reduce negative points and highlight suitable aspects.

People are only one group of the environmental beneficiaries. So, considering plants and animals during industrial activities is a necessity. Air and water pollution, soil erosion, and destroying rivers, seas, oceans, jungles and the other environmental elements are the negative results of development of human activities. All industries have their own share in this huge destruction and damage to the environment which must be minimized using new methods and technologies. Thus, disposing pollutions far away from the environment is required. In this regard, some operators consider the area of disposed pollutions as a sustainable resource which has this duty to prevent the environment from being infected by these troublous materials [2], such as concrete blocks for recycling waste soil and also solid parts (ores) of the waste coal slurries as cement material mixed with water $[3,4]$.

Oil and gas industry, same as the other fields, is on the way of changing and optimization to rescue the environment during its different and numerous projects. The important point about this sector compared to other ones is using natural resources in this industry. Therefore, petroleum scientists have a significant role in preservation and protection of the environment. In this area, one of the most related things is water. The way oil and gas companies meet water challenges is a serious subject. One of the optimum methods in oil and gas industry to deal with water scarcity issue is water reuse. Activities in oil and gas fields cause effluents that can be harmful for soil, water, air and other environmental components [1]. Thus, taking proper action about such water is a need, and reusing this water not only is an appropriate method for preventing environmental pollution, but is also helpful in water management policies.

As it is reported by Guerra et al. [2], for utilizing produced water of oil and gas industry reusing this wastewater or converting it to new water are two important ways. But to reach this aim, various parameters must be considered such as the geologic zone that this water is produced and will be used, the chemical and geographical aspects, and type of the produced hydrocarbon. In addition, many minerals or extra materials are dissolved in produced water which varies according to the location and type of soil and hydrocarbons. Bicarbonate, chloride, sodium, benzene, toluene, and other chemicals with various concentrations can be dissolved in water. The water physical properties like temperature vary depending on well type and situation. Based on these characteristics, materials and their amounts, and considering water and soil physical and chemical properties the water can be used for various applications such as crop irrigation, animal husbandry and industrial usages. Most of the mentioned accompanying materials, will remain in soils and that is why water can be harmful to the environment-in both short-term and longterm uses.

In addition to reusing solution, wastewater as one of the byproducts of petroleum industry is usually disposed to subsurface via injection wells [5]. Besides, induced seismicity and other limitations in subsurface disposal are existing problems which are the subject of many research studies in this field. To solve underground wastewater disposal problems, desalination - as an effort to achieve sustainability-is 
used by many authorities [6, 7]. Also, it is necessary to manage this method by making and implementing suitable rules, improving the quality of produced water, and facing with economic issues appropriately [5].

Furthermore, many studies have been accomplished in order to reach a safe water disposal approach which is appropriate both economically and environmentally. Considering the mentioned relation between seismic events and water disposal activities, predicting such events can provide a more optimized water disposal operation. On this way, it is necessary to analyze and study various kinds of parameters such as heterogeneity, size, pressure, porosity, conductivity, compressibility, fluid type, natural fractures (NFs) of the host reservoir layer. Therefore, following a smart well monitoring can improve disposal well activities. Such method tries to monitor the injection behavior in real time considering geology and petrophysical data and evidences [8].

The other proper approach for underground disposal of produced water is injecting the wastewater into the aquifers [9]. Using this method, similar to water disposal into oil and gas reservoirs, the water is injected to the deep subsurface geologic structures. One the one hand, according to the study by Raju et al. [10], the produced water in oil and gas wells, includes dissolved gasses such as $\mathrm{H} 2 \mathrm{~S}, \mathrm{CO} 2$ and also $\mathrm{CaCO} 3$ as well as sulfide sediments. On the other hand, the aquifer waters bear iron around $2 \mathrm{mg} / \mathrm{L}$. Thus, the combination of these two, can damage the rock structure. But according to the research studies in this area, this damage can be limited by initial permeability of the aquifer's rock [10]. As a matter of fact, this damage is not expected in more permeable aquifer rocks.

As another important subject about water in oil and gas industry, investigating around hydraulic fracturing wastewater issue is one of the hot topics. The process of discharging wastewater of the hydraulic fracturing industry from subsurface can be dangerous for both human and other environmental members and components [11]. So, keeping this kind of wastewater far away from the surface of the earth and also fresh water resources may be the best way of excluding them from the environment. In addition, as research studies show that the major part of wastewater disposal takes place via injection wells [12], wastewater injection via hydraulically fractured wells can be an option for the operators as well. The crucial unknown part is analyzing how this underground injection can have influences on the quality of surface water, humans and other creatures' health, and environmental status.

Among different types of reservoir rocks, tight gas reservoirs (TGRs) are of unconventional petroleum reservoirs the most outstanding feature of which is the presence of tight pore throats in them. As a matter of fact, petroleum reservoir rocks are porous media which are constructed with two parts-solid or rock volume plus pore volume. The index of quantifying pore volume in a porous medium is porosity degree and it is defined as the ratio of pore volume to total volume of that porous medium (rock in this study). Also, fluid conductivity of a porous rock is quantified by permeability. Regarding the Darcy's law permeability is the correlating coefficient of fluid velocity and pressure gradient along the porous medium whose unit is $\mathrm{m}^{2}$ in the metric system and Darcy or milli-Darcy in the oilfield system. TGRs are mostly naturally fractured causing them to have two distinct porous media-matrix and fracture. A schematic view of naturally fractured reservoirs (NFRs) is shown in Figure 1 below. In this figure, both real and dual porosity model NFR schematics are shown and compared with each other. Dual porosity model is a simplistic model which considers NFs uniformly distributed in rock matrix space. Dual porosity model is used in the simulation of NFR TGR in this study. 


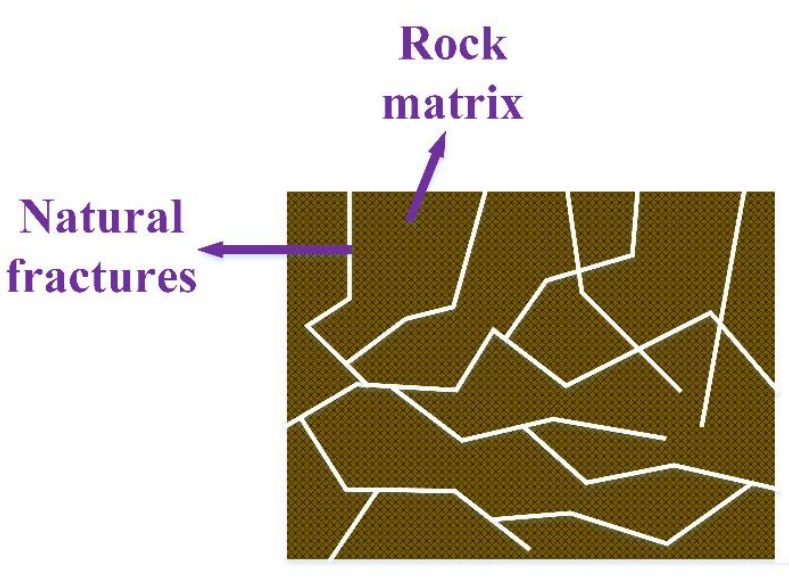

Real NFR

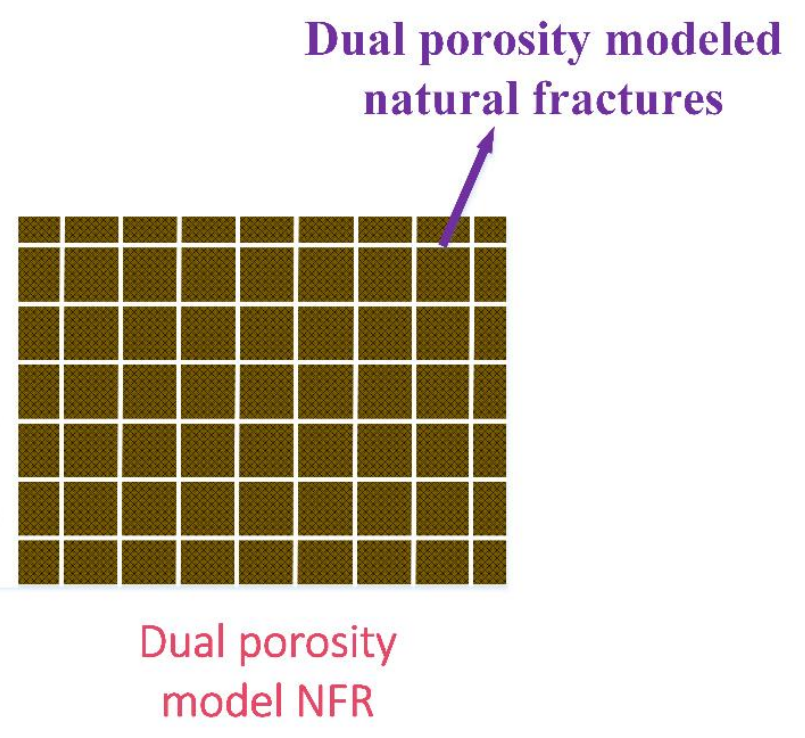

Dual porosity modeled natural fractures

Figure 1 Schematic picture of naturally fractured reservoirs consisting matrix blocks and fractures

As it is figured out by Schmidt et al. [13], increasing the bottomhole flowing pressure (BHFP) or increasing the rock permeability by reservoir stimulation, can provide improvement in the performance of saltwater injectivity. In limited geographic points, it is observed that there are increases in reservoir pressure as a result of the lower injection rates and also the higher average injection pressures. Thus, the geologic and geographic information must be considered during this type of investigation.

In tight and shale gas reservoirs, usual technique for production is different with conventional reservoir due to their poor reservoir quality (porosity and permeability) [14]. In these unconventional reservoirs, exploitation is applicable by drilling multistage fractured horizontal wells (MSFHWs). In fact, tight nature of these reservoirs makes the operators to implement rock stimulation using hydraulic fracturing to create several transverse hydraulic fractures (HFs) on the horizontal well for having more permeable pathways in these porous media [15]. This kind of reservoir stimulation makes the rock volume limited to the HFs have a better reservoir quality (higher porosity and permeability) compared to the unstimulated reservoir space. Thus, the stimulated reservoir space can be called stimulated rock volume (SRV), and reservoir space far away from the HFs can be named unstimulated rock volume (USRV).

Taking advantage of MSFHWs as a suitable technique for reservoir stimulation has been popular for a decade, especially in tight and ultra-tight hydrocarbon reservoirs to improve productivity of the wells. For the purpose of having an efficient recovery from unconventional petroleum resources with huge lateral extent compared to conventional reservoirs, taking advantage of horizontal wells is an inevitability [16]. On the one hand, horizontal wells have some benefits such as creating longer contact with reservoir surface, distributing pressure drops alongside the wellbore, and minimizing the chance of forming condensation in wellbore. On the other hand, horizontal completion merely fails to produce hydrocarbons in profit-making level due to the extremely low permeability of unconventional reservoirs such as TGRs. Hence, multistage hydraulic fracturing of horizontal wells for developing TGRs reservoir is used for resolving this problem [17]. Performance of a MSHFW, which is more complex than that of conventional wells, has to be analyzed for 
further model characterizations. Various research works have been conducted for this purpose studying some aspect of MSHFWs role in petroleum industry. Reservoir modeling is one of the most significant parts of these studies in which some useful information can be obtained from simulation results under various circumstances. Another topic in this area is local grid refinement (LGR). LGR method is one of the most popular techniques to define HFs in a numerical reservoir simulation model because it is easy to use and well accepted in the literature for representing the performance of hydraulically fractured wells in numerical simulation models $[18,19]$. As a matter of fact, locally refined grids in this approach explicitly demonstrate the main HFs [20]. The most convenient way to define LGR in a simulation model is to use small rectangular grid type, but the real shape of HFs is usually elliptical. Although the rectangular shape of the fractures cannot exactly emulate the real fracture geometry, it is a simple, applicable, and acceptable method to define HFs in a simulation model [21]. To improve the accuracy of real HFs' shape definition in the models, using combination of different LGRs with different sizes is preferred.

Size of the grid blocks in an LGR depends on the purpose of the simulation job. The reasonable grid size can be designed by using the results of history matching and well test reports to verify the accurate production prediction of the constructed model $[18,22]$. Some studies suggested different ways to create LGRs in simulation models such as Cartesian LGR in which grid sizes change alongside the various directions [18], cylindrical LGR that mostly encompasses near-wellbore illustrating for detailed pressure distributions [23], and some other types such as hexagonal, curvilinear, hybrid Cartesian, and hybrid-hexagonal LGRs [24].

In this study, a wastewater injection into a depleted TGR by a MSFHW is studied using numerical simulation. Eclipse commercial reservoir simulator is used for the numerical analysis. This reservoir simulator takes advantage of finite difference method (FDM) for numerical solution. In the base model reservoir, initial fluid is low-pressure methane gas with a little amount of connate water. Water injection is performed under constant BHFP condition via a 5-stage MSFHW (horizontal well with 5 transverse fractures). As the literature less approached engineering analyses of the rock properties and their effect on the performance of water injection, we analyze some properties of the TGR rock which stores the wastewater and investigate this kind of stimulated bed-rocks as sustainable resources for wastewater storage. Rock properties such as permeability and porosity of the rock matrix, NFs, and HFs are analyzed and their impacts on the water injection performance are studied and compared.

\section{Model Description}

To perform this study, a numerical reservoir model is constructed in the Eclipse commercial reservoir simulator. This reservoir model has a box-shaped structure with specified dimensions in $x, y$, and $z$ directions indicated in Table 1. Also, as it is determined in Table 1, initial condition of the reservoir shows that average pressure in whole reservoir grid blocks is 700 psia which is a low amount of pressure due to being a depleted reservoir. Number of reservoir grid blocks in $x, y$, and $z$ directions are $79 \times 19 \times 11$, respectively, and total reservoir size in each of $x, y$, and $z$ directions are shown in Table 1. Grid blocks which are locally refined in $\mathrm{x}$ and $\mathrm{y}$ directions vary logarithmically in size, but each of them has constant size of 20 feet in $z$ direction.

\section{Table 1 Base case reservoir data}




\begin{tabular}{|l|l|}
\hline Property & Value \\
\hline Initial pressure & $700 \mathrm{psia}$ \\
\hline Reservoir temperature & $200^{\circ} \mathrm{F}$ \\
\hline X dimension & $4926.58 \mathrm{ft}$ \\
\hline Y dimension & $4747 \mathrm{ft}$ \\
\hline Z dimension (reservoir thickness) & $220 \mathrm{ft}$ \\
\hline BHFP & $5000, \mathrm{psia}$ \\
\hline Matrix porosity & 0.09, fraction \\
\hline Fracture porosity & 0.005, fraction \\
\hline Matrix horizontal permeability & $0.5, \mathrm{md}$ \\
\hline Fracture horizontal permeability & $50, \mathrm{md}$ \\
\hline Matrix block size & $100, \mathrm{ft}$ \\
\hline Fracture-matrix transfer shape factor & $0.0012,1 / \mathrm{ft}^{2}$ \\
\hline Number of hydraulic fracture stages & 5 \\
\hline Hydraulic fracture permeability & 30000, md \\
\hline Hydraulic fracture porosity & 0.3, fraction \\
\hline Matrix block size & $100, \mathrm{ft}$ \\
\hline Initial gas saturation for matrix & 0.8, fraction \\
\hline Initial gas saturation for fracture & 0.99, fraction \\
\hline Initial water saturation for matrix & 0.2, fraction \\
\hline Initial water saturation for fracture & 0.01, fraction \\
\hline
\end{tabular}

Base case reservoir model has no SRV and all porosity and permeability values for matrix blocks and NFs are constant values as determined in Table 1. Also, HFs are defined in numerical model with a narrow width (0.016 feet) and constant permeability of 30000 md for base case model. Schematic view of numerical models of base case reservoir is depicted in Figure 2. The reservoir model shown in this figure varies in depth as it is demonstrated by different colors. Total amount of this depth variation is equal to total reservoir thickness (220 ft). It has to be declared that the used LGR for defining the HFs and horizontal well resembles the one applied in the study by Shirbazo et al. [18, 25].

Petrophysical and fluid properties of the synthetic TGR of this study have been selected with respect to other similar TGRs across the world. For instance, as the stored fluid in TGRs is mainly methane [26, 27], entire reservoir model of this study is considered to be saturated with pure methane. Also, amount of permeability and porosity for TGRs are mostly less than $0.1 \mathrm{md}$ and $10 \%$, respectively [28]. However, it is probable for these reservoirs to have permeability values on the order of $1 \mathrm{md}$ [26], such as Travis Peak formation-Texas, United States - with up to near $10 \mathrm{md}$ matrix permeability [29, 30]. Matrix permeability and porosity of base SGR in this study are $0.5 \mathrm{md}$ and 9\%, respectively. In addition, petrophysical properties of NFs have been selected in conformity with the similar TGRs properties available in the literature [3135]. Permeability of NFs can be in a range of near-matrix permeability values up to the order of 10 or even $100 \mathrm{D}$. Fracture porosity values are mainly in a range of near $0.2 \%$ to more than $1 \%$. NF permeability and porosity for the base reservoir model of this study are $50 \mathrm{md}$ and $0.5 \%$, respectively.

In addition, it has to be noted that horizontal permeability values for all reservoir grid block are equal in $\mathrm{x}$ and $y$ directions $\left(k_{x}=k_{y}=k_{h}\right)$. However, vertical permeability values for all grid blocks are one tenth of horizontal permeability $\left(\mathrm{k}_{\mathrm{z}}=0.1 \mathrm{k}_{\mathrm{h}}\right)$. 


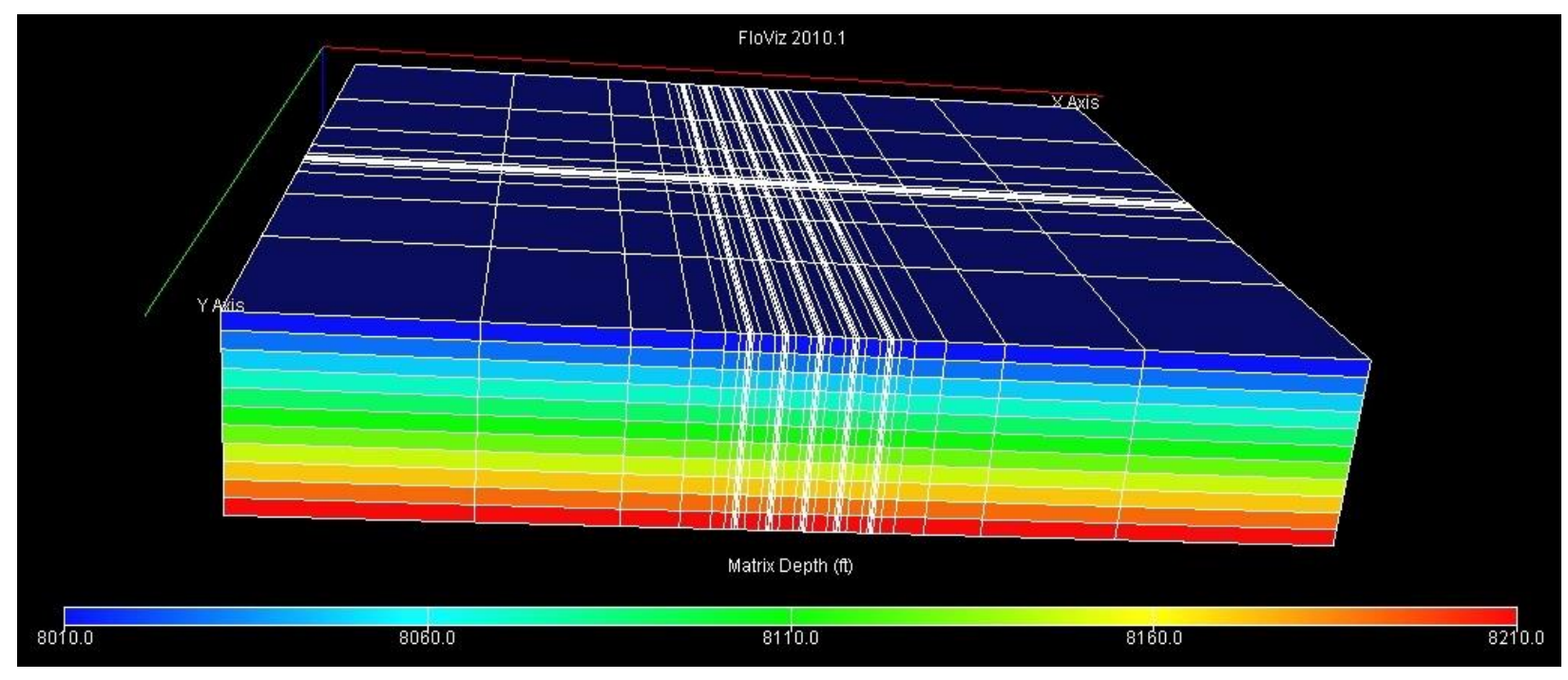

Figure 2 Schematic view of base case reservoir numerical model

In order to analyze the constructed reservoir model for its response to water injection under different circumstances several cases for matrix blocks, NFs, and HFs property variation are considered. As the first case of analysis-declared in Table 2-porosity and permeability of both matrix and NF media of SRV are set to be higher than that of USRV (base case model) with three different values to see their effect on the results for volume of injected water. Moreover, as the second analysis, a sensitivity analysis of HFs permeability for volume of injected water results is designed for further investigations. Table 2 shows all variable properties values used in sensitivity analyses.

Table 2 Variability of fractured rock properties for sensitivity analysis

\begin{tabular}{|l|l|l|}
\hline Case & Property & Value \\
\hline$\# 1$ & SRV matrix horizontal permeability & $1,2,4 \mathrm{md}$ \\
\hline$\# 2$ & SRV NF horizontal permeability & $100,200,400 \mathrm{md}$ \\
\hline$\# 3$ & SRV matrix porosity & $0.108,0.126,0.162$, fraction \\
\hline$\# 4$ & SRV NF porosity & $0.006,0.007,0.009$, fraction \\
\hline$\# 5$ & HF permeability & $15000,40000,50000 \mathrm{md}$ \\
\hline
\end{tabular}

\section{Results}

By running the simulation of the water injection to the base case TGR model via a MSFHW for 123 days, the results for the total volume of the injected water and also field pressure are depicted in Figure 3. As it is depicted in this figure, within 123 days of water injection, cumulative water injection will be finally reached to less than 14 billion barrels, and field pressure will be increased to near 860 psia due to water injection for the base case model. 


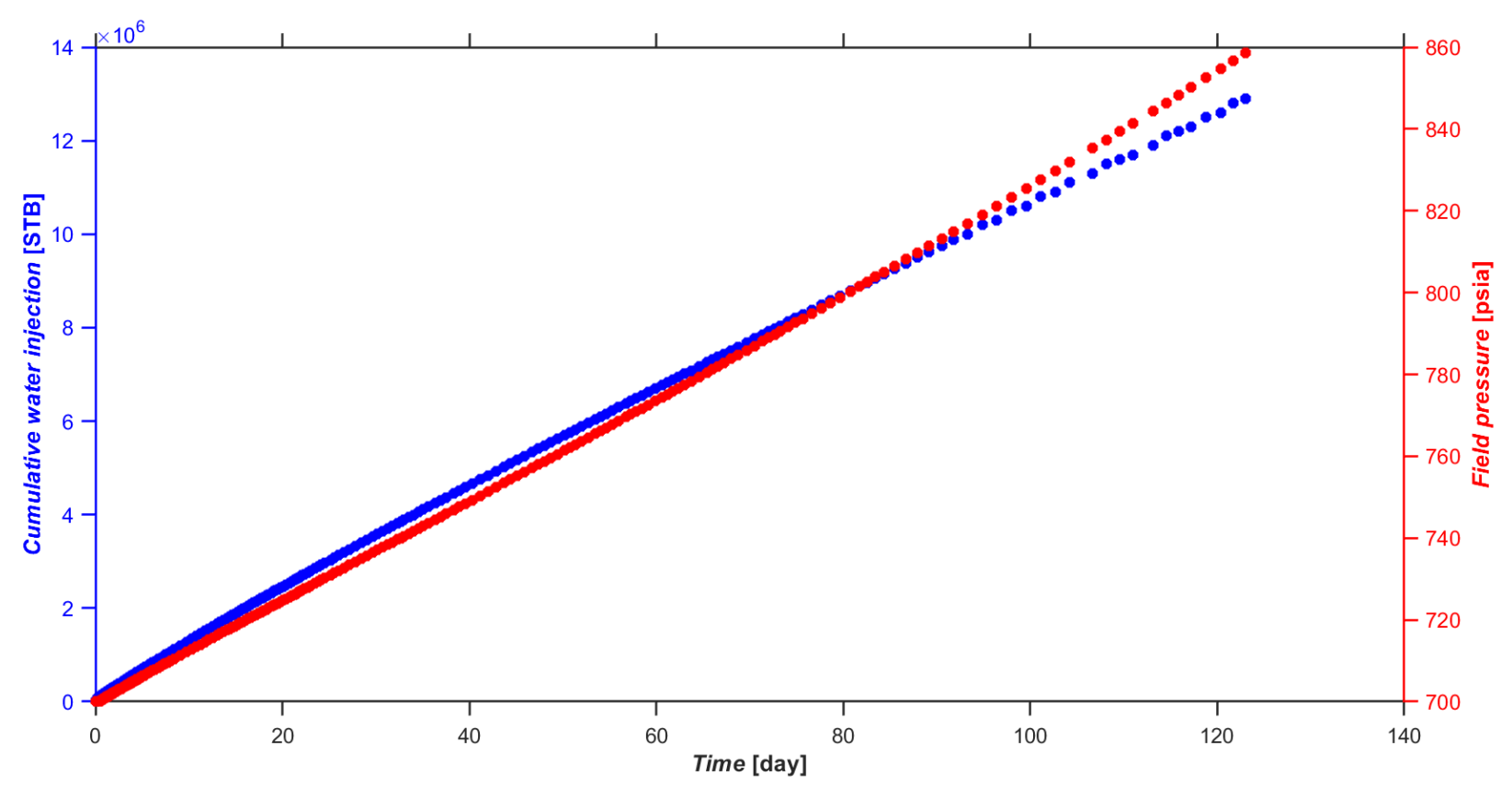

Figure 3 Base case reservoir field cumulative water injection and pressure

For analyzing importance of taking into account SRV reservoir properties in performance of wastewater injection into the reservoir via a MSFHW, sensitivity of simulated cumulative water injection to four SRV properties are analyzed. These analyses are performed for matrix permeability, NF permeability, matrix porosity, and NF porosity in which these properties are designed to have specific amount in SRV as a multiplier of USRV properties values. Amount of these four properties is clarified in Table 1 for base case model (USRV), and Table 2 for sensitivity analyses for SRV properties. Figure 4 below shows the result of 123-day simulation for the cases 1 to 4 of Table 2 within a-d figures, respectively. 

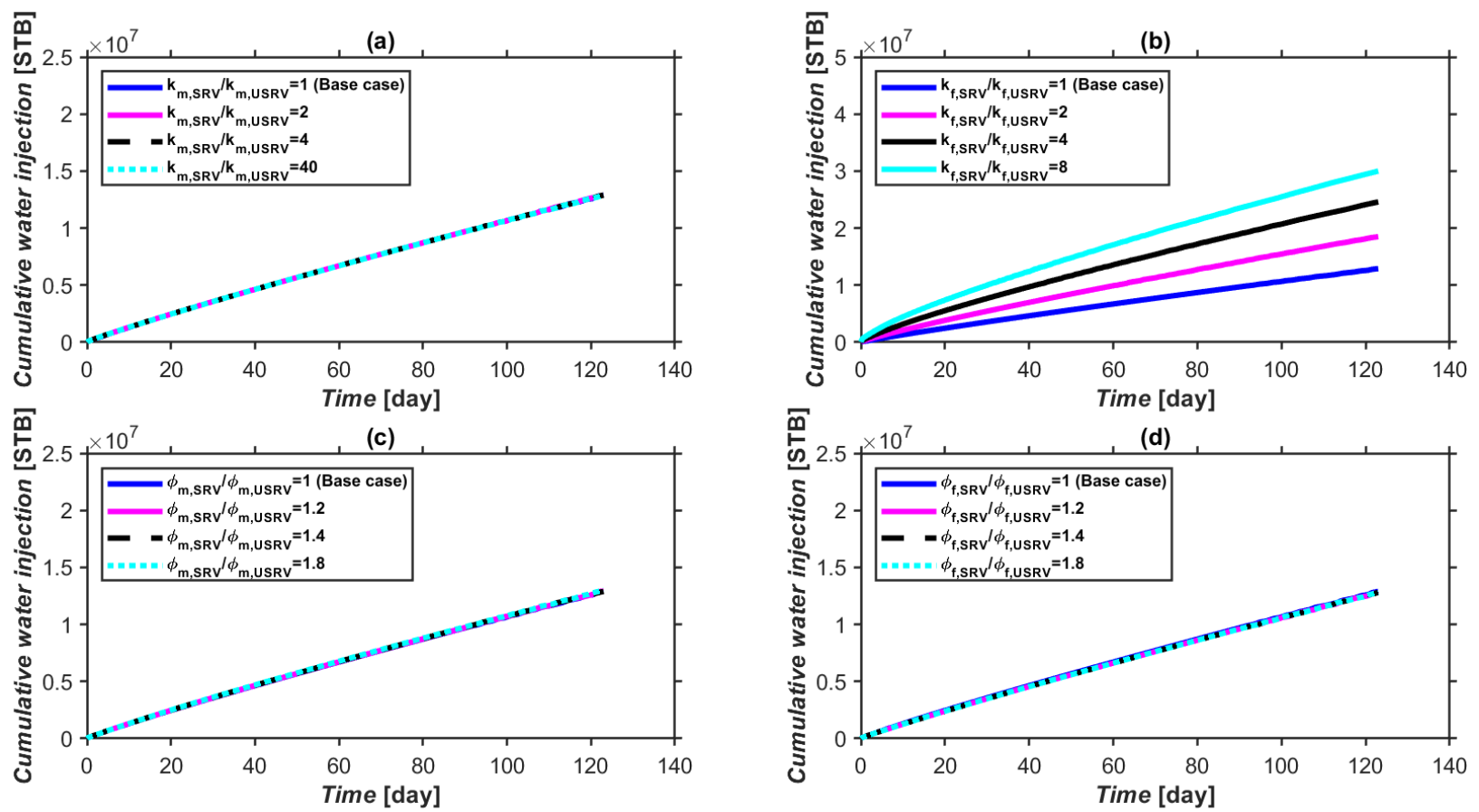

Figure 4 Field cumulative water injection into the base case reservoir with sensitivity to SRV's rock properties: matrix permeability (a), fracture permeability (b), matrix porosity (c), and fracture porosity (d)

It can be inferred from Figure 4 that this simulation is not sensitive to three properties of naturally fractured SRVs; matrix permeability, matrix porosity, and NF porosity. However, variation in NF permeability significantly affects total volume of injected water to the host TGR. Obviously, the more NF permeability is, the more cumulative water injection amount will be.

Furthermore, by running the simulation considering different amounts for HF permeability for 123 days, results are shown in Figure 5. As it is recognizable from this figure, by an increase in HF permeability values cumulative water injection increases. 


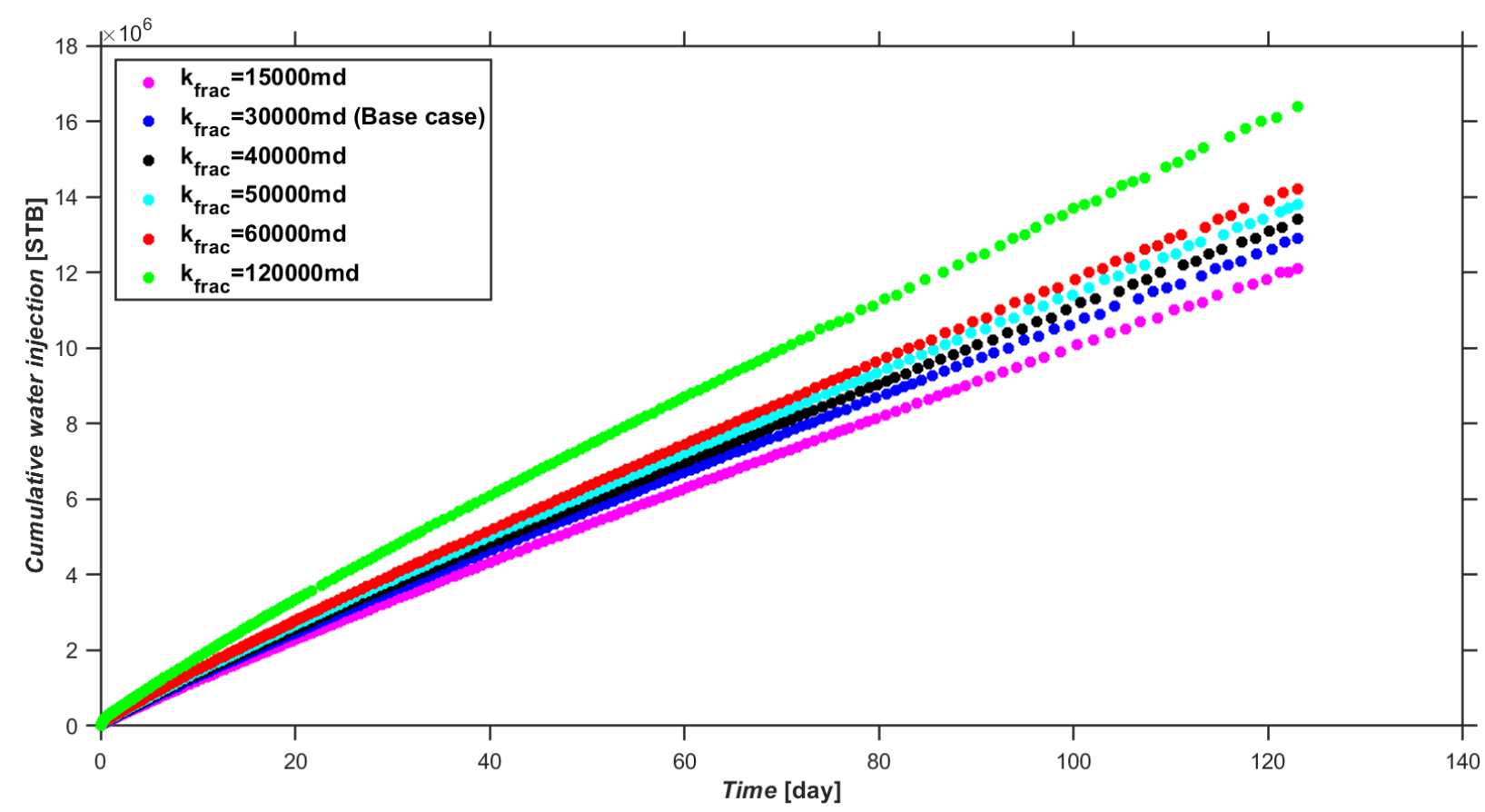

Figure 5 Field cumulative water injection into base case reservoir with sensitivity to hydraulic fracture permeability

The results taken from analysis of Figures 4 and 5 make sense according to this statement that effect of permeability on flow efficiency of a porous medium is much greater than effect of porosity of that porous medium. Among permeability of three different media in the studied TGR-matrix, NF, and HF permeability-different effects of them are analyzed. It can be implied from Figures $4 \mathrm{a}$ and $4 \mathrm{~b}$ that how increase in permeability of the matrix and NF, respectively, can change the total water injection response. On the one hand, it can be inferred from Figure 4a that total water injection to the TGR cannot be changed by increasing matrix permeability of SRV even up to the case SRV matrix permeability is 40 times the USRV matrix permeability. On the other hand, cumulative water injection shows a considerable sensitivity to NF permeability in Figure $4 \mathrm{~b}$. In this figure, the more SRV to USRV NF permeability is, the more total water volume is injected-and stored-in the TGR.

By further analysis, it can be concluded from Figure $4 \mathrm{~b}$ that as NF permeability of SRV becomes 2-, and 4time higher than NF permeability of USRV, average cumulative water injection at the end of the simulation period will be 18.5 and 24.6 MMSTB. As the final amount of cumulative water injection for base case is 12.9 MMSTB, it means that in cases with NF permeability of SRV 2-, and 4-time higher than NF permeability of USRV total response of water injection will be increased almost $43 \%$ and $90 \%$, respectively. In addition, in different cases of HF permeability (Figure 5), HF permeability 60000 and 120000 md have final amounts for cumulative water injection of 14.2 and 16.4 MMSTB, respectively. In other words, as HF permeability becomes 2-, and 4-time higher than base case HF permeability total injected water volume to the TGR will be increased near $10 \%$ and $27 \%$, respectively. Thus, it can be concluded that NF permeability is technically a more effective parameter than HF permeability in total storage response of water injection into the TGR. Further economic studies can be derived with this approach that investing on the reservoirs with higher NF permeability can be more beneficial than on the technologies creating more conductive HFs. 


\section{Conclusion}

A numerical simulation model of a TGR has been created in this work. Simulation study was a wastewater injection into the reservoir porous media to store the disposed water far away from the earth's surface. Water injection carries out via a MSFHW with $5 \mathrm{HF}$ stages. By running the simulation over an approximately 4-month period of water injection, two important findings are obtained. First, it is concluded that total volume of injected water is sensitive to both NF and HF permeability with a positive correlation, and it cannot be considerably affected by other petrophysical properties of fractured media such as matrix permeability, matrix porosity, and fracture porosity. In addition, it is eventuated that cumulative water injection is more sensitive to NF permeability change than the variation in HF permeability.

\begin{tabular}{ll}
\multicolumn{2}{l}{ Acronyms } \\
BHFP & Bottomhole flowing pressure \\
CaCO3 & Calcium carbonate \\
CO2 & Carbon dioxide \\
D & Darcy \\
H2S & Hydrogen sulfide \\
HF & Hydraulic fractures \\
LGR & Local grid refinement \\
md & Milli-Darcy \\
MMSTB & Million stock-tank barrels \\
MSFHW & Multi-stage fractured horizontal well \\
NF & Natural fracture \\
NFR & Naturally fractured reservoir \\
SRV & Stimulated rock volume \\
STB & Stock-tank barrels \\
TGR & Tight gas reservoir \\
USRV & Unstimulated rock volume
\end{tabular}

\section{References}

[1] A. Davarpanah: "Feasible analysis of reusing flowback produced water in the operational performances of oil reservoirs", Environmental Science and Pollution Research, vol. 25, pp. 35387-35395, 2018.

[2] K. Guerra, K. Dahm, and S. Dundorf: "Oil and Gas Produced Water Management and Beneficial Use in the Western United States", Washington, DC , 2011.

[3] J.A. Ferriz-Papi and S. Thomas: "Recycled Aggregates from Construction and Demolition Waste in the Production of Concrete Blocks", Journal of Construction Materials, vol. 2, no. 1, 2020.

[4] F. Sartipi and A. Sartipi: "Recycling of coal mining slurry in concrete mortar", Journal of Construction Materials, vol. 1, no. 1, 2019. 
[5] B.R. Scanlon, R.C. Reedy, P. Xu, M. Engle, J.P. Nicot, D. Yoxtheimer, Q. Yang, and S. Ikonnikova: "Can we beneficially reuse produced water from oil and gas extraction in the U.S.?", Science of the Total Environment, vol. 717, 2020.

[6] D.L. Shaffer, L.H. Arias Chavez, M. Ben-Sasson, S. Romero-Vargas Castrillón, N.Y. Yip, and M. Elimelech: "Desalination and reuse of high-salinity shale gas produced water: Drivers, technologies, and future directions" Environmental Science and Technology, vol. 47, no. 17, pp. 9569-9583, 2013.

[7] K. Jiang, H. Chamani, T. Matsuura, D. Rana, and C. Lan: "Effect of temperature and salt on PVDF membrane wetting properties" Journal of Construction Materials, vol. Special Issue on Sustainable Materials in Petroleum and Chemical Engineering , 2021.

[8] M.P. Gogri, J.M. Rohleder, C.S. Kabir, M.J. Pranter, and Z.A. Reza: "Prognosis for safe waterdisposal-well operations and practices that are based on reservoir flow modeling and real-time performance analysis" SPE Reservoir Evaluation and Engineering, vol. 21, no. 03, pp. 576-592, 2018.

[9] R. McCurdy: "Underground Injection Wells For Produced Water Disposal", 2011. https://www.epa.gov/sites/production/files/documents/21_McCurdy_-_UIC_Disposal_508.pdf.

[10] K.U. Raju, H.A. Nasr-El-Din, V. V. Hilab, S. Siddiqui, and S. Mehta: "Injection of aquifer water and gas/oil separation plant disposal water into tight carbonate reservoirs", SPE Journal, vol. 10, no. 04, pp. 374-384, 2005.

[11] W.D. Burgos, L. Castillo-Meza, T.L. Tasker, T.J. Geeza, P.J. Drohan, X. Liu, J.D. Landis, J. Blotevogel, M. McLaughlin, T. Borch, and N.R. Warner: "Watershed-Scale Impacts from Surface Water Disposal of Oil and Gas Wastewater in Western Pennsylvania", Environmental Science and Technology, vol. 51, no. 15, pp. 8851-8860, 2017.

[12] C.D. Kassotis, L.R. Iwanowicz, D.M. Akob, I.M. Cozzarelli, A.C. Mumford, W.H. Orem, and S.C. Nagel: "Endocrine disrupting activities of surface water associated with a West Virginia oil and gas industry wastewater disposal site", Science of the Total Environment, vol. 557-558, pp. 901-910, 2016.

[13] D. Schmidt, A.C.M. Miguens, C. Wong, K. Hlava, and D. Pinkston: "Salt water disposal performance in the Williston basin", SPE Western Regional Meeting Proceedings, 2019.

[14] A. Taghavinejad, M. Sharifi, E. Heidaryan, K. Liu, and M. Ostadhassan: "Flow modeling in shale gas reservoirs: A comprehensive review", Journal of Natural Gas Science and Engineering, vol. 83, pp. 103535, 2020.

[15] S. Dahim, A. Taghavinejad, M. Razghandi, H. Rahimi Rigi, K. Moeini, S. Jamshidi, and M. Sharifi: "Pressure and Rate Transient Modeling of Multi Fractured Horizontal Wells in Shale Gas Condensate Reservoirs", Journal of Petroleum Science and Engineering, vol. 185, no. February 2020, pp. 106566, 2020.

[16] A. Satter and G.M. Iqbal: "Oil and gas recovery methods in low permeability and unconventional reservoirs" Reservoir Engineering: The Fundamentals, Simulation, and Management of Conventional and Unconventional Recoveries. pp. 353-364. Gulf Professional Publishing, 2016.

[17] K. Al-Garadi, A. Aldughaither, M. Ba Alawi, H. Al-Hashim, N. Sibaweihi, and M. Said: "A novel approach for optimizing multistage hydraulic fracturing of gas condensate horizontal wells" SPE Middle East Oil and Gas Show and Conference, MEOS, Proceedings, 2019. 
[18] A. Shirbazo, J. Fahimpour, and B. Aminshahidy: "A new approach to finding effective parameters controlling the performance of multi-stage fractured horizontal wells in low-permeability heavy-oil reservoirs using RSM technique", J. Pet. Explor. Prod. Technol., vol. 10, pp. 3569-3586, 2020.

[19] K. AlObaidi: "Induced Fractures Modelling in Reservoir Dynamic Simulators", Institute of Petroleum Engineering, Heriot Watt University, 2014.

[20] X. Xue, C. Yang, T. Onishi, M.J. King, and A. Datta-Gupta: "Modeling hydraulically fractured shale wells using the fast marching method with local grid refinements LGRs and embedded discrete fracture model EDFM", Society of Petroleum Engineers - SPE Reservoir Simulation Conference, 2019.

[21] S.S. Abdelmoneim, A.M. Shehata, M. Kamel, and A. Aly: "Modeling hydraulic fractures in finite difference simulators using amalgam LGR (Local Grid Refinement)", Society of Petroleum Engineers - North Africa Technical Conference and Exhibition 2012, NATC 2012: Managing Hydrocarbon Resources in a Changing Environment (2012).

[22] C.W.J. Berentsen, J.R. Shaoul, C.J. De Pater, J. Park, P. Dabrowski, S. Dobkine, O. Khudyakova, A. Nesterenko, and I. Yushkov: "An efficient method for explicit hydraulic fracture representation in full field reservoir simulation”, Proceedings - SPE Annual Technical Conference and Exhibition, 2014.

[23] N. Bhore: "An investigation on qualitative analysis of hydraulic fracture simulation for field development planning", SPE Annu. Tech. Conf. Exhib. Society of Petroleum Engineers, 2017.

[24] K. Aziz: "Reservoir simulation grids:opportunities and problems", JPT, Journal of Petroleum Technology, vol. 45, no. 07, pp. 658-663fr, 1993.

[25] A. Shirbazo, A. Taghavinejad, and S. Bagheri: "CO2 Capture and Storage Performance Simulation in Depleted Shale Gas Reservoirs as Sustainable Carbon Resources", Journal of Construction Materials, vol. Special Issue on Sustainable Materials in Petroleum and Chemical Engineering, 2021.

[26] J.G. Speight: "Properties Processing of Gas From Tight Formations", Deep Shale Oil and Gas. pp. 307-347. Elsevier, 2017.

[27] N.B. Harris, T. Ko, R.P. Philp, M.D. Lewan, C.J. Ballentine, Z. Zhou, and D.L. Hall: "Geochemistry of Natural Gases from Tight- Gas-Sand Fields in the Rocky Mountains. Report No. 07122-09, National Energy Technology Laboratory, Unites States Department of Energy, Washington, DC" , 2013.

[28] A. Bahadori: "Overview of Natural Gas Resources", Natural Gas Processing. pp. 1-22. Elsevier, 2014.

[29] S.A. Holditch: "Tight gas sands", Journal of Petroleum Technology, vol. 58, no. 06, pp. 86-93, 2006.

[30] M.A. Fracasso, S.P. Dutton, and R.J.. Finley: "Depositional Systems and Diagenesis of Travis Peak Tight Gas Sandstone Reservoirs, Sabine Uplift Area, Texas", SPE Formation Evaluation, vol. 3, no. 01, pp. 105-115, 1998.

[31] H. Bahrami, R. Rezaee, and M. Hossain: "Characterizing natural fractures productivity in tight gas reservoirs", Journal of Petroleum Exploration and Production Technology, vol. 2, no. 2, pp. 107-115, 2012.

[32] J.E. Olson, S.E. Laubach, and R.H. Lander: "Natural fracture characterization in tight gas sandstones: Integrating mechanics and diagenesis", AAPG Bulletin, vol. 93, no. 11, pp. 1535-1549, 2009.

A. Taghavinejad et al. /Journal of Construction Materials | Special Issue 2021 
[33] J.E. Olson, S.E. Laubach, and P. Eichhubl: "Estimating natural fracture producibility in tight gas sandstones: Coupling diagenesis with geomechanical modeling", Leading Edge (Tulsa, OK), vol. 29, no. 12, pp. 1494-1499, 2010.

[34] C. Freeman, G.J. Moridis, G.E. Michael, and T.A. Blasingame: "Measurement, Modeling, and Diagnostics of Flowing Gas Composition Changes in Shale Gas Wells", SPE Latin America and Caribbean Petroleum Engineering Conference., Mexico City, Mexico, 2012.

[35] G.J. Moridis, T.A. Blasingame, and C.M. Freeman: "Analysis of mechanisms of flow in fractured tight-gas and shale-gas reservoirs", SPE Latin American and Caribbean Petroleum Engineering Conference Proceedings. Society of Petroleum Engineers, 2010. 